\title{
Effect of Different Solution Flow Rates on Analyte Ion Signals in Nano-ESI MS, or: When Does ESI Turn into Nano-ESI?
}

\author{
Andrea Schmidt and Michael Karas \\ Institute for Pharmaceutical Chemistry, Johann Wolfgang Goethe University, Frankfurt a.M., Germany
}

Thomas Dülcks

Department of Biology and Chemistry, University of Bremen, Bremen, Germany

In nano-ESI MS, the qualitative and quantitative characteristics of mass spectra vary considerably upon the use of different spraying conditions, i.e., aperture of the spraying needle and the voltage applied. The major parameters affected by the aperture size is the liquid flow rate which determines the initial droplet size and the current emitted upon the spray process, as described by different models of the ESI process. In the present study, the effect of flow rate on ion signals was studied systematically using mixtures of compounds with different physicochemical properties (i.e., detergent/oligosaccharide and oligosaccharide/peptide). For these model systems, the functional dependence of certain analyte-ion ratios upon the flow rate can be correlated to changes in analyte partition during droplet fission prior to ion release. Analyte suppression is practically absent at minimal flow rates below $20 \mathrm{~nL} / \mathrm{min}$. (J Am Soc Mass Spectrom 2003, 14, 492-500) (C) 2003 American Society for Mass Spectrometry

$\mathrm{E}$ lectrospray ionization mass spectrometry (ESI MS) [1-3] has become a powerful tool in modern bioanalytics and drug research and is used nowadays a routine analytical method [4-7]. However, although ESI MS is applied routinely for a variety of analytical investigations, there are still cases that point out the limits of this technique with respect to an efficient analyte ionization and thus analyte detection. For instance, the analysis of hydrophilic compounds is still not possible with good sensitivity using high-flow rate techniques. Furthermore, contaminations in the analyte solution such as salts or detergents, or the presence of other analytes, may lead to substantial suppression of the analyte signal [8]. Within the last years, nanoelectrospray (nano-ESI) developed by Wilm and Mann [9] has found a broad application in many MS laboratories. It employs spray needles pulled from glass capillaries to a fine tip with o.d. in the micrometer range. Since the flow rates induced by the electrospray process (without external pumping) are in the range of about some tens of $\mathrm{nL} / \mathrm{min}$, just a few $\mu \mathrm{L}$ of analyte solution suffice for extended mass spectrometric studies.

Besides the fact that only a low amount of analyte solution is required for nano-ESI MS investigations, it

Published online April 7, 2003

Address reprint requests to Dr. A. Schmidt, Institute for Pharmaceutical Chemistry, Johann Wolfgang Goethe University, Marie-Curie-Str. 97, D-60590 Frankfurt a.M., Germany. E-mail: Andrea.Schmidt@iachem.de has been shown that purely aqueous solutions can be sprayed without the need of any organic dopant [10], and that hydrophilic compounds such as carbohydrates, can be detected with high sensitivity without chemical derivatization, which is usually applied when using high flow-rate ESI [11]. Furthermore, nano-ESI has been shown to be more tolerant towards salt contaminations in the analyte solution than conventional ESI [10, 12].

The advantages of nano-ESI in comparison to the high-flow rate techniques can be rationalized based on the fundamentals of the ESI process. A lower solution flow rate is known to reduce the size of the charged droplets initially produced in the spraying process. Different approaches exist to describe this fact, i.e., by Wilm and Mann [9], Fernandez de la Mora et al. [13], and Pfeifer and Hedricks [14]. The smaller initial droplets require fewer droplet fission events and less solvent evaporation prior to ion release into the gas phase. As a consequence, a larger portion of the analyte molecules present in the primary droplets is made available for MS analysis, and the increase in concentration of contaminants is significantly reduced. Thus, nano-ESI may be considered to be "more than just a miniaturized" conventional ESI source [12]. However, it is known to practitioners of nano-ESI that absolute signal intensities obtained for a given analyte solution or signal intensity ratios obtained for given mixtures may vary significantly upon the use of different nano-ESI needles, which makes quantitation experiments difficult or even 
impossible. The analysis of mixtures, especially those which consist of compounds with very different physico-chemical properties, is often a challenge in ESI MS. Ion signals of hydrophilic analytes are easily suppressed by surface active contaminants of the solution. This surface activity can be either "chemical" (e.g., caused by solvophobic parts of the molecule) or induced by a suitable charge which drives the ion towards the droplet surface. A quantitative treatment of the analyte-suppression effect is given by the surface partition model developed by Enke and co-workers [15-17]. Surface activity is an important factor which influences the tendency of a compound to reach the next droplet generation upon an asymmetric fission event. The enrichment of surface-active compounds in the progeny droplets has been shown recently [18].

The often poor reproducibility of signal intensities from analyte mixtures in nano-ESI points to a more fundamental problem which is connected to different shapes and opening diameters of the spray tips and also results from the typical handling procedure of the nano-ESI needles. If the needle aperture is too small to immediately initiate the spray, or if needle clogging occurs during the analysis, the tip is usually gently touched against the counter electrode to break it up. As a consequence, the final tip opening diameters may vary considerably even in cases where identical nanoESI needles were used initially. The aperture size is one important parameter affecting the liquid flow rate, which in turn determines the size of the primary droplets generated in the spraying process $[9,13,14]$. Therefore, the question is addressed whether the advantages of "true" nano-ESI-i.e., the capability of sensitive analysis of hydrophilic compounds, higher tolerance towards salt contaminations and less analyte suppression effects in mixtures-are really valid for all nano-ESI needles and all nano-ESI conditions. If the special properties of nano-ESI depend on the solution flow rate, a transition should exist between nano-ESI and conventional ESI, which is mainly dependent on the tip opening diameter, and which should be measurable by a gradual loss of the nano-ESI properties with increasing flow rate. In the present study, we describe systematically the effect of the tip diameter (and hence the flow rate) in nano-ESI. To this end, we have chosen suitable model compounds which allow for a qualitative comparison of the generated droplet sizes. Furthermore, we have investigated the effect on the analysis of mixtures which usually exhibit strong analyte suppression effects, such as mixtures of hydrophilic compounds with those which exhibit a pronounced surface activity.

\section{Experimental}

Nano-ESI needles of various tip diameters were pulled from borosilicate glass capillaries (Clark Electromedical Instruments, Pangbourne, U.K., $1.2 \mathrm{~mm}$ o.d., $0.65 \mathrm{~mm}$ i.d.) with a micropipette puller (Model P-97, Sutter
Instrument Co., Novato, CA). The tip size was determined with a microscope equipped with a calibrated ruler in one ocular with a precision of approximately \pm $1 \mu \mathrm{m}$. Tip outer diameters of $1-2.5 \mu \mathrm{m}$ and above $20 \mu \mathrm{m}$ could be obtained by adjusting the pulling parameters. Tips with o.d. between 2.5 and $15 \mu \mathrm{m}$ were produced from $1 \mu \mathrm{m}$ tips which were gently touched against a glass plate to break them up. After optical inspection under a microscope, all tips were coated with gold by means of a sputter coater (Model K 550, Emitech Ltd, Ashford, U.K.).

ESI MS experiments were performed on an orthogonal-acceleration time-of-flight instrument (oTOF MS, Mariner PE/PerSeptive Biosystems, Framingham, MA) equipped with a nano-ESI source (Protana, Odense, DK). The atmospheric pressure-vacuum interface was modified from the original nozzle-curtain gas setup to a heated transfer capillary setup (150 mm in length, 0.5 $\mathrm{mm}$ i.d.) [19]. The spray current (typically in the nanoampere range) was measured by an additional, lab-built floatable amperometer. Instrumental settings were the following : $\mathrm{U}_{\text {transfer-cap/skimmer }}=10-300 \mathrm{~V}, \mathrm{U}_{\text {skimmer/ }}$ transfer-quad $=6-12 \mathrm{~V}$. Mass spectra were recorded in positive-ion mode by averaging 10 single spectra with $3 \mathrm{~s}$ acquisition time each. Based on several random samples, the spectrum-to-spectrum fluctuation of signal intensity ratios was determined to be in the order of $10 \%$. This value was used to indicate the confidence of each data point. Empirical fitting of the data points was accomplished by the Microcal Origin V 6.0, Microcal Software Inc. Northampton, MA.

All analytes were purchased from Sigma-Aldrich Chemie GmbH, Taufkirchen, Germany and used without further purification. Solvents (HPLC grade) and acetic acid (glacial, 100\%) were from Carl Roth $\mathrm{GmbH}$, Karlsruhe, Germany. Barium bromide was dissolved in ethanol at a concentration of $\mathrm{c}=10^{-4} \mathrm{~mol} / \mathrm{L}$. The mixture of turanose (Scheme 1, $1, \mathrm{M}=342.3 \mathrm{~g} / \mathrm{mol}$ ) and n-octyl-glucopyranoside $(\underline{2}, \mathrm{M}=292.4 \mathrm{~g} / \mathrm{mol})$ was prepared in methanol/0.1 mM aqueous sodium chloride (vol:vol 30:70) with the analytes in concentrations of $10^{-5} \mathrm{~mol} / \mathrm{L}$ and $10^{-6} \mathrm{~mol} / \mathrm{L}$, respectively. Mixtures of maltoheptaose $(\mathrm{M}=1152.0 \mathrm{~g} / \mathrm{mol})$ and neurotensin $(\mathrm{M}=1672.9 \mathrm{~g} / \mathrm{mol})$ were prepared either in methanol/ water/acetic acid (vol:vol 48:48:4) in concentrations of $10^{-4}$ and $10^{-5} \mathrm{~mol} / \mathrm{L}$, respectively, or in methanol/10 $\mathrm{mM}$ aqueous ammonium acetate (vol:vol 1:1) with both analytes present in an equimolar concentration of $10^{-5}$ $\mathrm{mol} / \mathrm{L}$.

\section{Flow Rate Determination}

To determine the flow rate, 5 to $10 \mu \mathrm{l}$ of analyte solution were loaded into the different nano-ESI needles. The weight of the filled needles was measured on a fine balance (Type 2405, Sartorius GmbH, Göttingen, Germany). The nano-ESI needles were then mounted in front of the mass spectrometer and the spray voltage was carefully raised to start the spray process without 


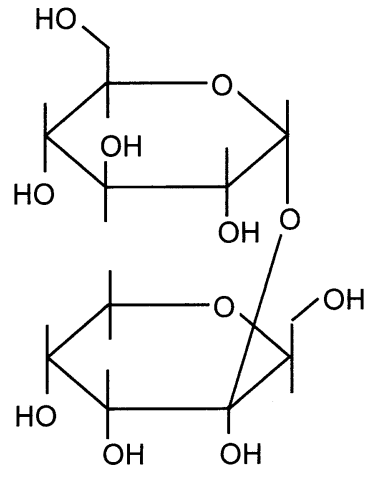

1

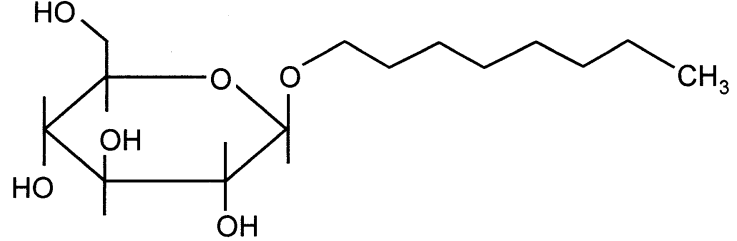

2

Scheme 1.

inducing corona discharge as assessed by the current measurement. Supply of the analyte solution to the spray tip was achieved without any external pumping or application of back pressure. The spray was maintained for $15 \mathrm{~min}$ for all capillaries under investigation, monitored by the spray current and the mass spectra. After the spraying period, the weight of the capillary was measured again and the mass of solution consumed was corrected by the amount of solvent that usually evaporates during the weighing and handling procedure, as determined in separate "blank" experiments. The actual volume of sample solution consumed during the spraying process was calculated from the corrected mass by using the density of the respective solvents. The error for each flow rate data point is estimated by the range of values obtained for the "blank" experiments ( $\pm 1.5 \mathrm{nl} / \mathrm{min})$

\section{Optical Observation of the Spray Process}

The nano-ESI needles were mounted at a distance of 2-3 $\mathrm{mm}$ from a grounded metal plate which served as the counter electrode. A voltage between 800 and $1000 \mathrm{~V}$ was applied to the needle and the spray process was monitored by the current between the needle and the counter electrode. The needle tip was observed with a stereomicroscope. Photographs were taken through one ocular with a digital camera.

\section{Results and Discussion}

The mass spectra of analyte mixtures obtained by nano-ESI MS analysis may exhibit significant differences upon the use of different nano-ESI needles. This phenomenon is observed in particular if the compounds present in the mixture differ considerably in those properties which are known to affect their ionization efficiency in ESI MS, such as surface activity or charge $[8,16,20]$. As an example Figure 1 shows the mass spectra of an equimolar mixture of neurotensin and maltoheptaose in neutral solution obtained before (Figure 1a) and after (Figure 1b) breaking up the capillary tip by gently touching the counter electrode. In the first case ion signals of both analytes, [neurotensin $+2 \mathrm{H}]^{2+}$ and [maltoheptaose $+\mathrm{Na}^{+}$, are detected in nearly equal intensities, whereas in the second case neurotensin is clearly dominating. The signal intensities observed in different experiments (i.e., different capillaries and different opening procedures) may vary, as the experimental conditions in the opening procedure are only ill defined, but the general trend as shown in Figure 1 remains unchanged. It is an obvious conclusion that the major difference between the spray before and after breaking up the capillary tip is the increase of liquid flow rate obtained for the larger tip opening, and in association with this, other properties of the spray such as the initial droplet size and the spray current undergo substantial changes. Furthermore, also the field strength at the tip may change due to the change in field focusing. The experiments described in the following aim to correlate the spectral characteristics of indicative analyte mixtures to the determined flow rates.

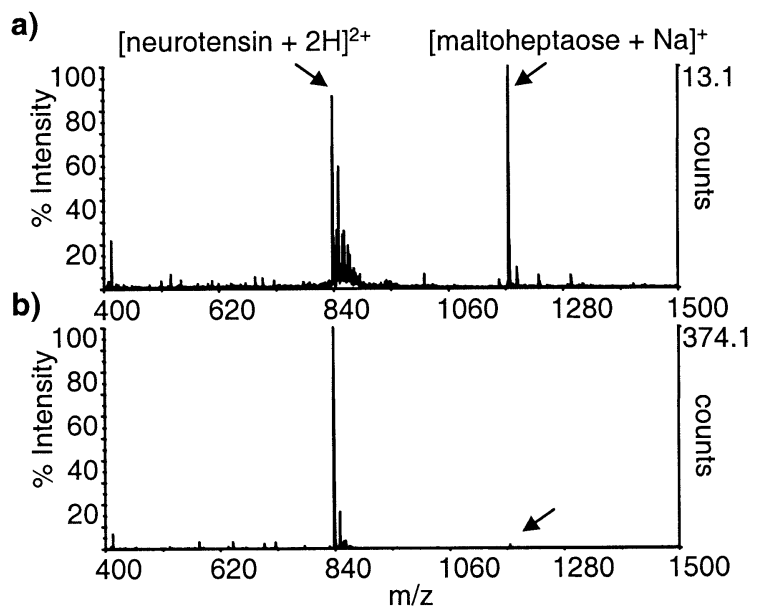

Figure 1. Nano-ESI mass spectra of an equimolar mixture (c = $10^{-5} \mathrm{~mol} / \mathrm{L}$ ) of neurotensin and maltoheptaose in methanol/10 $\mathrm{mM}$ aqueous ammonium acetate (vol:vol 1:1), average of 10 spectra at $3 \mathrm{~s}$ each. The spray current was constant over the time of spectra acquisition. (a) capillary tip o.d. $<1 \mu \mathrm{m}$. (b) after breaking up the tip, o.d. $\approx 10 \mu \mathrm{m}$. 
a)

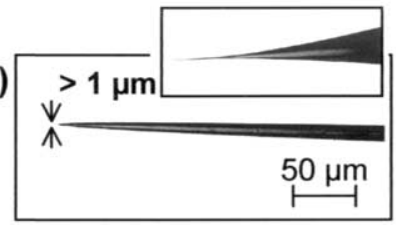

c)

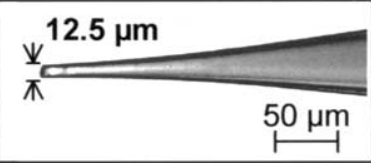

b)

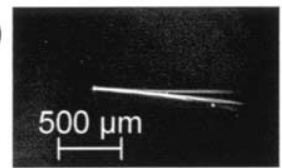

d)

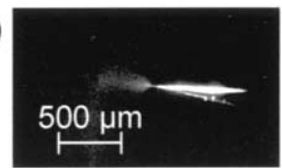

Figure 2. Photographs of nano-ESI spray capillaries taken through the ocular of a microscope. The spray was observed for a mixture of methanol/water/acetic acid (48:48:4). (a) spray tip o.d. $<1 \mu \mathrm{m}$; the overall shape of the capillary is shown in the insert with 10-fold lower magnification. (b) $>1 \mu \mathrm{m}$ o.d. capillary, no visible spray at measurable spray current. (c) spray tip o.d. 12.5 $\mu \mathrm{m}$. (d) $12.5 \mu \mathrm{m}$ capillary, visible spray.

\section{Flow Rate, Droplet Size, and Spray Current}

In order to monitor the initial differences in the spraying conditions, nano-ESI needles with tip diameters between $\approx 1$ and $30 \mu \mathrm{m}$ were inspected optically during the spray process. A current in the range of 10 to $100 \mathrm{nA}$ between the needle and the counter electrode was indicative of the onset of the spray. The photographs obtained for two tip diameters, $\approx 1$ and $12.5 \mu \mathrm{m}$, are shown in Figure 2. It turned out that only for tip diameters above $2.5 \mu \mathrm{m}$ (photograph not shown) can the spray be observed visually as a fine mist of droplets. This observation can either be attributed to a higher amount of solvent sprayed (and therefore an increase in droplet number), or to the formation of larger initial droplets.

Several models exist to predict the changes in initial droplet radius $R_{\text {ini }}$ upon variation of the liquid flow rate $\mathrm{V}_{\mathrm{fl}}$, as summarized in Table 1. These models allow for an estimate of the initial droplet size generated by nano-ESI depending on solution properties. The theoretical droplet sizes at the typical nano-ESI liquid flow rate of $20 \mathrm{~nL} / \mathrm{min}$ are also summarized in Table 1. Other parameters needed for the calculation (see leg-

end) are chosen for the typical nano-ESI analysis of a methanol/water mixture containing a few percent of acetic acid: $\rho=933.65 \mathrm{~kg} / \mathrm{m}^{3}$ [21], $\varepsilon=49.4$ [22], $\gamma=$ $0.03531 \mathrm{~N} / \mathrm{m}$ [23], $\mathrm{K}=0.1 \mathrm{~S} / \mathrm{m}, \mathrm{E}=7,52 \cdot 10^{7} \mathrm{~V} / \mathrm{m}$ (calculated as discussed in reference [24]), $\mathrm{U}_{\mathrm{T}}=550 \mathrm{~V}$, $\mathrm{U}_{\mathrm{a}}=600 \mathrm{~V}$. Under these assumptions the calculation according to the model of Pfeifer and Hendricks yields primary droplet radii in the $100 \mu \mathrm{m}$ range which exceed by orders of magnitude the droplet sizes observed in the experiment. Thus, calculations based on this model should be treated with caution, as mentioned earlier [24]. On the other hand, the droplet radii obtained with the models of Fernandez de la Mora and Wilm both lie in the sub-micrometer range which is in better accordance to the optical observations.

Flow rates were investigated for a mixture of methanol and $10 \mathrm{mM}$ aqueous ammonium acetate (vol:vol 1:1) spayed from capillaries of different o.d.. As shown in Figure 3a, the flow rates are in the range between 20 and $600 \mathrm{~nL} / \mathrm{min}$, however, there is generally only a rough correlation between flow rate and tip o.d.. A better correlation was obtained between the liquid flow rate and the current emitted during the spraying process, which is shown in Figure 3b. The observed behavior significantly differs from the current-flow rate scaling laws of Pfeifer, $\mathrm{I} \propto \mathrm{V}_{\mathrm{fl}}^{4 / 7}$, and Fernandez de la Mora, $\mathrm{I} \propto \mathrm{V}_{\mathrm{fl}}^{1 / 2}$, (see Table 1), however, for this particular solvent system the correlation with an empirical function $V_{\mathrm{fl}} \propto \mathrm{I}^{5.5}$, determined by fitting the experimental data to the equation $\mathrm{V}_{\mathrm{fl}}=\mathrm{P} 1+\mathrm{P} 2 \cdot \mathrm{I}^{\mathrm{P3}}\left(\mathrm{V}_{\mathrm{fl}}\right.$ : flow rate, I: current, P1, P2, P3: fitting parameters), yielded a good fit.

Obviously, the spray current does not rise linearly with the flow rate. The spray current, i.e., the number of excess charge carriers results from electrochemical reactions upon the spray process, which include the discharge of counterions and redox reactions of the solvent that can lead to changes in solution $\mathrm{pH}$ when aqueous solutions are used [25]. Solvent electrolysis will be even more pronounced at high current densities at the spray tip which is achieved under the usual nano-ESI conditions [26]. The effect on solvent compo-

Table 1. Summary of the different scaling laws for the size of initial droplets at different liquid flow rates

\begin{tabular}{lccc}
\hline & Pernandez de & $\begin{array}{c}\text { F } \\
\text { la Mora and } \\
\text { Loscertales } \\
{[13]}\end{array}$ & Wilm and Mann [9] \\
\hline \hline Equation & $\mathrm{R}=\left(\frac{3 \varepsilon \gamma^{1 / 2} \mathrm{~V}_{\mathrm{fl}}}{4 \pi \varepsilon_{0}^{1 / 2} \mathrm{KE}}\right)^{2 / 7}$ & $\mathrm{R} \propto\left(\frac{\mathrm{V}_{\mathrm{fl}} \varepsilon \varepsilon_{0}}{\mathrm{~K}}\right)^{1 / 3}$ & $\mathrm{R} \propto\left(\frac{\rho}{4 \pi^{2} \gamma \tan (\pi / 2-\theta)\left(\left(\mathrm{U}_{\mathrm{T}} / \mathrm{U}_{\mathrm{a}}\right)^{2}-1\right)}\right)^{1 / 3} \mathrm{~V}_{\mathrm{fl}}^{2 / 3}$ \\
$\begin{array}{l}\text { Dependence between droplet } \\
\text { radius and flow rate }\end{array}$ & $\mathrm{R} \propto \mathrm{V}_{\mathrm{fl}}^{2 / 7}$ & $\mathrm{R} \propto \mathrm{V}_{\mathrm{fl}}^{1 / 3}$ & $\mathrm{R} \propto \mathrm{V}_{\mathrm{fl}}^{2 / 3}$ \\
$\begin{array}{l}\text { Dependence between current } \\
\text { and liquid flow rate }\end{array}$ & $\mathrm{I} \propto \mathrm{V}_{\mathrm{fl}}^{4 / 7}$ & $\mathrm{I} \propto \mathrm{V}_{\mathrm{fl}}^{1 / 2}$ & $\mathrm{na}$ \\
$\begin{array}{l}\text { Calculated droplet radius for } \\
\mathrm{V}_{\mathrm{fl}}=20 \mathrm{~nL} / \mathrm{min}\end{array}$ & $1.4 \cdot 10^{-4} \mathrm{~m}$ & $1.1 \cdot 10^{-7} \mathrm{~m}$ & $7.7 \cdot 10^{-8} \mathrm{~m}$ \\
\hline
\end{tabular}

Abbreviations: $\gamma$ : surface tension of the solvent, $\varepsilon$ : permittivity of the solvent, $\varepsilon_{0}$ : permittivity of the vacuum, K: conductivity of the solution, E: electric field at the capillary tip, $\rho$ : density of the liquid; $\theta$ : liquid cone angle (classical Taylor cone theory: $\theta=49.3^{\circ}$ ), $U_{\mathrm{T}}$ : theshold voltage, $\mathrm{U}_{\mathrm{a}}$ : applied voltage. 


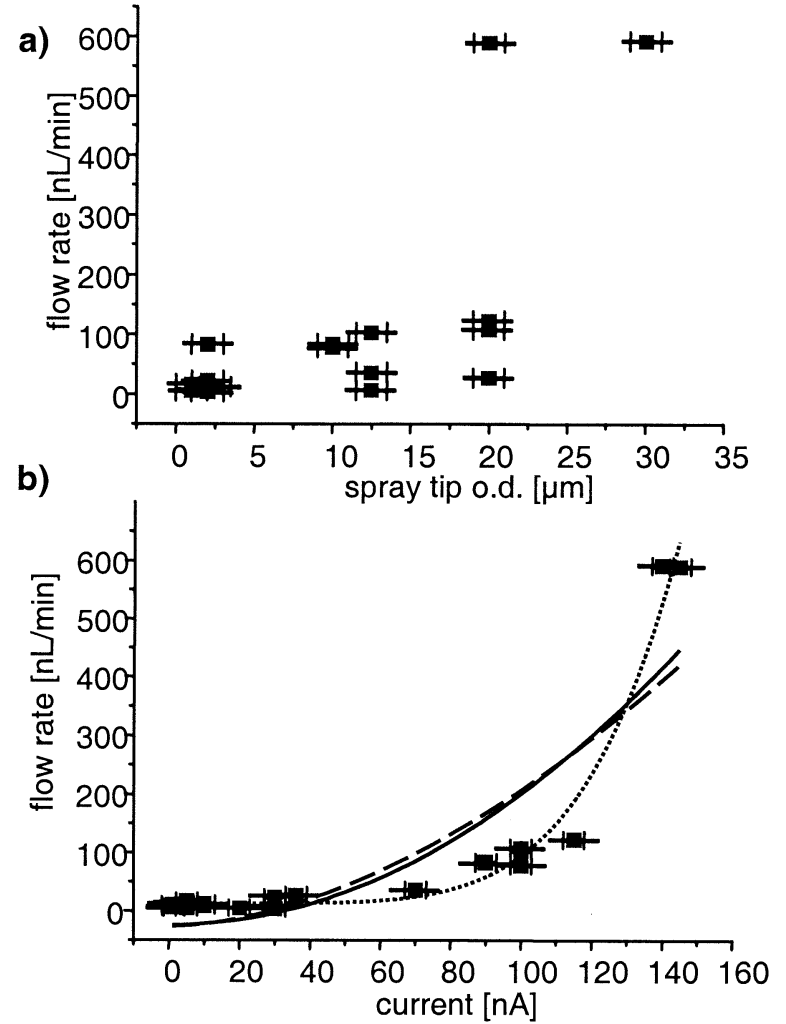

Figure 3. Flow rates obtained for a mixture (vol:vol 1:1) of methanol/10 mM ammonium acetate in water. (a) as a function of spray tip opening, (b) as a function of spray current. Solid line: Fernandez de la Mora and Loscertales, $\mathrm{V} \propto \mathrm{I}^{2}$ [13]; dashed line: Pfeifer and Hendricks, $\mathrm{V} \propto \mathrm{I}^{7 / 4}$ [14]; dotted line: empirically fitted function, $\mathrm{V} \propto \mathrm{I}^{5.5}$.

sition can be estimated by calculation of the concentration of excess charge carriers $[Q]$ connected with the spray current $\mathrm{I}$ at a certain flow rate $\mathrm{V}_{\mathrm{fl}}$ [27]:

$$
[\mathrm{Q}]=\mathrm{I} /\left(\mathrm{V}_{\mathrm{fl}} \cdot \mathrm{F}\right)
$$

where $\mathrm{F}=$ Faraday constant $\left(9.648 \cdot 10^{4} \mathrm{C} \mathrm{mol}^{-1}\right)$. The values for four experimental data points are summarized in Table 2. The data show that electrochemical reactions are more pronounced at low flow rates while the effect becomes less significant when flow rates comparable to conventional ESI are approached. The resulting concentration of charge carriers is less than proposed previously [27], since the measured current is

Table 2. Amount of excess charge carriers ${ }^{\mathrm{a}}$

\begin{tabular}{lcc}
\hline Spray current $[\mathrm{nA}]$ & Flow rate $[\mathrm{nL} / \mathrm{min}]$ & {$[\mathrm{Q}][\mathrm{mol} / \mathrm{L}]$} \\
\hline \hline 10 & 9 & $6.91 \cdot 10^{-4}$ \\
30 & 23 & $8.11 \cdot 10^{-4}$ \\
115 & 122 & $5.86 \cdot 10^{-4}$ \\
145 & 589 & $1.53 \cdot 10^{-4}$ \\
\hline
\end{tabular}

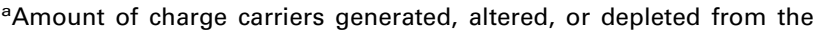
analyte solution at different measured flow rates and spray currents for a mixture of methanol/10 mM aqueous ammonium acetate (vol:vol 1:1). Data was taken from Figure 3

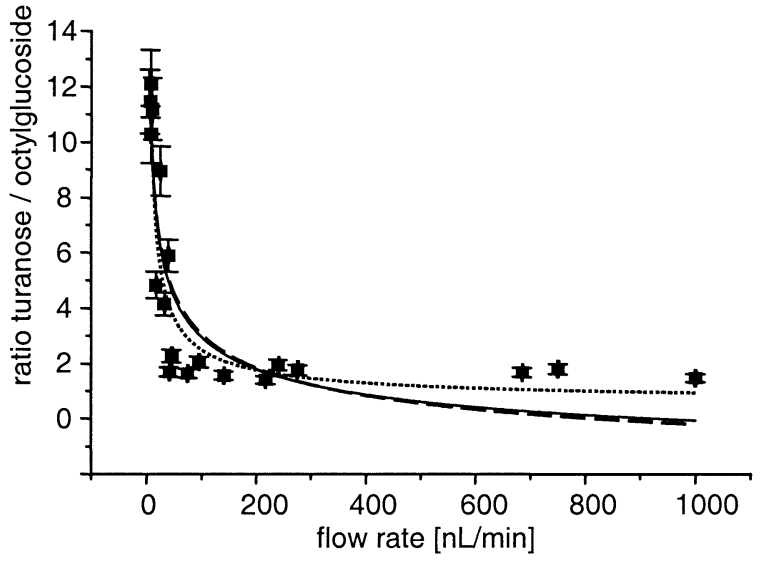

Figure 4. Signal intensity ratios of the sodiated molecular ions obtained for a mixture of n-octyl-glucopyranoside (c $=10^{-6}$ $\mathrm{mol} / \mathrm{L})$ and turanose $\left(\mathrm{c}=10^{-5} \mathrm{~mol} / \mathrm{L}\right)$ in methanol/water (30:70). Curves represent the changes in surface-to-volume ratio of the initial droplets upon variation of the flow rate according different models. Solid line: Fernandez de la Mora and Loscertales [13]; dashed line: Pfeifer and Hendricks [14]; dotted line: Wilm and Mann [9].

lower than estimated. Nevertheless, alterations in solvent composition should be considered as a further source for changes in analyte response at different liquid flow rates.

\section{Influence of Flow Rates on Nano-ESI Mass Spectra of Analyte Mixtures}

The influence of the flow rate on signal intensities of compounds with different chemical surface activity was studied using a mixture of turanose and n-octyl-glucopyranoside (see Scheme 1). Turanose as a typical disaccaride is hydrophilic, whereas n-octyl-glucopyranoside exhibits a pronounced surface activity because of its nonpolar n-octyl residue. Both analytes are only weak solution-phase acids or bases and therefore uncharged in solution. Thus, their affinity for the charged droplet surface under ESI conditions is only determined by their chemical surface activity and not affected by any solution-phase pre-charge. Both analytes are ionized by sodium-ion attachment upon their transfer from the charged droplets into the gas phase, thus, any alteration in solvent composition due to an altered extent of redox reactions influences their ion signal intensity in the same way.

The experiments were carried out using the carbohydrate in a 10-fold higher concentration to compensate for the strong suppression effects occurring at higher liquid flow rates. Figure 4 shows the ratio of signal intensities of turanose and n-octylglucopyranoside for different liquid flow rates. Even though the absolute signal intensities of both components rise with increasing spray current, there is a strong suppression of the disaccharide. At the minimal flow rates of a few $\mathrm{nL} /$ min signal suppression effects have totally disappeared while at flow rate above $50 \mathrm{~nL} / \mathrm{min}$ the suppression 
amounts to about a factor of five. There is even a slight preference for the disaccharide compared to the detergent at minimal flow rates which may reflect the higher cationization efficiency of a disaccharide compared to the derivatized monosaccharide.

The changes in analyte signal intensity ratios can be understood in terms of analyte partition upon droplet fission prior to ion release [18, 28]. A compound's surface activity is a crucial parameter determining its tendency to reach the next droplet generation in the droplet fission cascade, which is a prerequisite for its release into the gas phase. Larger initial droplets require higher number droplet fission events prior to ion release, thus, suppression of a hydrophilic analyte is more pronounced under these conditions. For a mixture of a hydrophilic and a surface-active analyte, the surface-tovolume ratio of the initial droplets affects the distribution of each analyte through the droplet fission cascade and, as a consequence, determines the relative signal intensities of both analytes in the mass spectra.

The surface-to-volume ratio of a spherical droplet is proportional to $1 / R$, and therefore the scaling laws for the surface-to-volume ratio in dependence of the flow rate are: $\propto 1 / \mathrm{V}_{\mathrm{fl}}^{2 / 7}$ for Pfeifer and Hendricks, $\propto 1 / \mathrm{V}_{\mathrm{fl}}^{1 / 3}$ for Fernandez de la Mora and Loscertales, and $\propto 1 / \mathrm{V}_{\mathrm{fl}}^{2 / 3}$ for Wilm and Mann, respectively. In order to assess a possible connection between the suppression of the hydrophilic analyte and the surface-to-volume ratio of the initial droplets, the experimental data points, i.e., signal intensity ratios, were fitted to the equation $y=P 1$ $+\left(\mathrm{P} 2 \cdot 1 / \mathrm{V}_{\mathrm{fl}}^{\mathrm{m}}\right)$ with $\mathrm{y}$, experimental data points $\mathrm{P} 1, \mathrm{P} 2$, variable fitting parameters, and $\mathrm{m}$, exponential factor according to the different scaling laws. The results reveal a clear correlation between the changes in signal intensity and the changes in liquid flow rate, and the closest correlation is obtained for the calculations according to Wilm and Mann.

Beside an analyte's chemical surface activity, its charge-derived surface activity in ESI droplets should also be taken into account as a parameter for analyte suppression effects. This question was addressed using mixtures of a peptide (neurotensin) and a carbohydrate (maltoheptaose) as representative system. In acidic solution, neurotensin is protonated because of solutionphase acid-base reactions, whereas maltoheptaose is uncharged due to the lack of any acidic or basic sites. Upon transfer into the gas phase, neurotensin is mainly detected as doubly and triply protonated molecular ions, whereas maltoheptaose is singly sodiated. The intensity ratio between the sodiated maltoheptaose ( $\mathrm{c}=$ $10^{-4} \mathrm{M}$ ) and the protonated molecular ions of neurotensin $\left(\mathrm{c}=10^{-5} \mathrm{M}\right.$, charge states +2 and +3$)$ is shown in Figure 5a. The more pronounced suppression of the hydrophilic carbohydrate can be rationalized by the higher surface activity of the peptide which is attributable to its chemical properties as well as due to its charge under the solution conditions investigated. The actual extent of protonation due to acid-base equilibrium reactions may alter on the way from the bulk

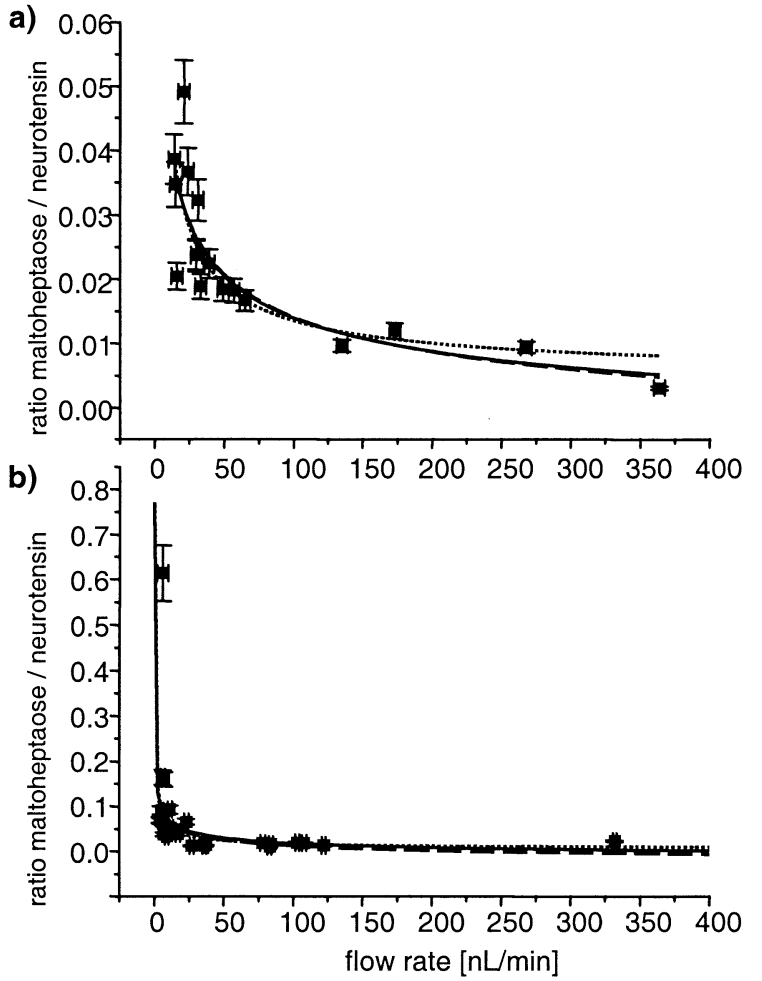

Figure 5. Signal intensity ratios between sodiated maltoheptaose and the sum of both protonated molecular ions of neurotensin, $[\text { Mhep }+\mathrm{Na}]^{+} /\left([\mathrm{N}+2 \mathrm{H}]^{2+}+[\mathrm{N}+3 \mathrm{H}]^{3+}\right)$, at different liquid flow rates. (a) maltoheptaose $10^{-4} \mathrm{~mol} / \mathrm{L}$, neurotensin $10^{-5}$ mol/L, solvent: methanol/water $/ 4 \%$ acetic acid. (b) equimolar mixture ( $\mathrm{c}=10^{-5} \mathrm{~mol} / \mathrm{L}$ ), solvent: methanol $/ 10 \mathrm{mM}$ aqueous ammonium acetate. The changes in surface-to-volume ratio of the initial droplets upon variation of the flow rate according different models are indicated. Solid line: Fernandez de la Mora and Loscertales [13]; dashed line: Pfeifer and Hendricks [14]; dotted line: Wilm and Mann [9].

solution into the gas phase because of alterations in solution-phase composition. Nevertheless, the peptide is positively charged and may therefore contribute to the surplus charge carriers located on the droplet surface in positive-ion mode. Maltoheptaose, on the other hand, has a low tendency towards the droplet surface, since this molecule is hydrophilic and uncharged in solution. The mixture therefore exhibits similar properties as described for the system turanose/n-octylglucopyranoside.

Also, effects resulting from changes in solution composition can influence the ion signals in this model system. As the extent of redox reactions is more pronounced at lower flow rates (Table 2), lower flow rates can either cause a relatively higher concentration of $\mathrm{H}_{3} \mathrm{O}^{+}$(caused by oxidation of the solvent), or a higher extent of anion depletion. It is believed that more than one type of redox reaction occurs [26]. However, a higher concentration of $\mathrm{H}_{3} \mathrm{O}^{+}$would favor the detection of protonated neurotensin at lower flow rates, while a higher extent of anion depletion should enhance the detection of both analytes in general. Thus, the observation that suppression of the hydrophilic analyte is 
reduced at low flow rates cannot be explained by these effects.

Figure $5 \mathrm{a}$ shows the fit of the analyte intensity ratio to the changes in droplet surface-to-volume ratio upon variation of the liquid flow rate (see above). The experimental data agree best with the $\propto 1 / \mathrm{V}_{\mathrm{fl}}^{2 / 3}$ scaling law predicted by the model of Wilm and Mann, pointing to the role of the surface-to-volume ratio in the initial droplets as a relevant parameter for analyte suppression in this model system.

The signal suppression effect observed in acidic solution for neurotensin and maltoheptaose is less pronounced if the analyte mixture is buffered with ammonium acetate $(\mathrm{pH} 7)$. Due to the higher $\mathrm{pH}$, the protonation equilibrium of neurotensin is shifted towards a lower solution-phase charge state. This is also reflected by a shift of the charge states in the mass spectra. In acidic solution, the ion signal intensity of the $3+$ charge state is higher than than that of the $2+$ charge state by a factor of approximately 15, while in the buffered solution the $2+$ charge state is higher by a factor of approximately 50 .

The absolute values of the intensity ratio for neurotensin and maltoheptaose in an equimolar mixture (both analytes: $\mathrm{c}=10^{-5} \mathrm{M}$ ) are in the range between 0.02 and 0.6 and are systematically higher than the ratios obtained in the acidic mixture which even contained a 10-fold excess of maltoheptaose (Figure 5a), indicating that a larger portion of the carbohydrate is made available for MS analysis. Consequently, suppression effects between neurotensin and maltoheptaose are reduced if the charge of the peptide due to solutionphase protonation is lowered by increasing the $\mathrm{pH}$ of the analyte solution. The ratio obtained at very low flow rates almost reflects the actual equimolar concentration in solution.

The results clearly indicate that the solution flow rate is crucial for the analysis of mixtures; moreover, advantages also exist for analyte solutions containing salt contaminations. This is shown as an example in Figure 6 for insulin in methanol/water $/ 4 \%$ acetic acid solution containing $1 \mathrm{mM} \mathrm{NaCl}$. The sample is analyzed at different liquid flow rates which are in this case monitored indirectly by measurement of the current emitted by the spray capillary. Under these solution-phase conditions, insulin is mainly detected in the charge states +4 up to +6 (Figure 6a). At a higher spray current $(60 \mathrm{nA})$ and therefore a higher liquid flow rate, the lower charge states show pronounced adduct formation with sodium ions, as shown in Figure $6 \mathrm{~b}$ for the charge state +4 . At a significantly reduced spray current $(\sim 1$ $\mathrm{nA}$ ) which is indicative of a lower liquid flow rate, adduct formation can be reduced or even completely prevented (Figure 6c). This can be either due to a lower extent of solvent evaporation and thus concentration of contaminants prior to ion release, or due to a higher extent of solvent oxidation and thus formation of $\mathrm{H}_{3} \mathrm{O}^{+}$ upon the spray process. The comparison of absolute signal intensities reveals that in the case of the lower

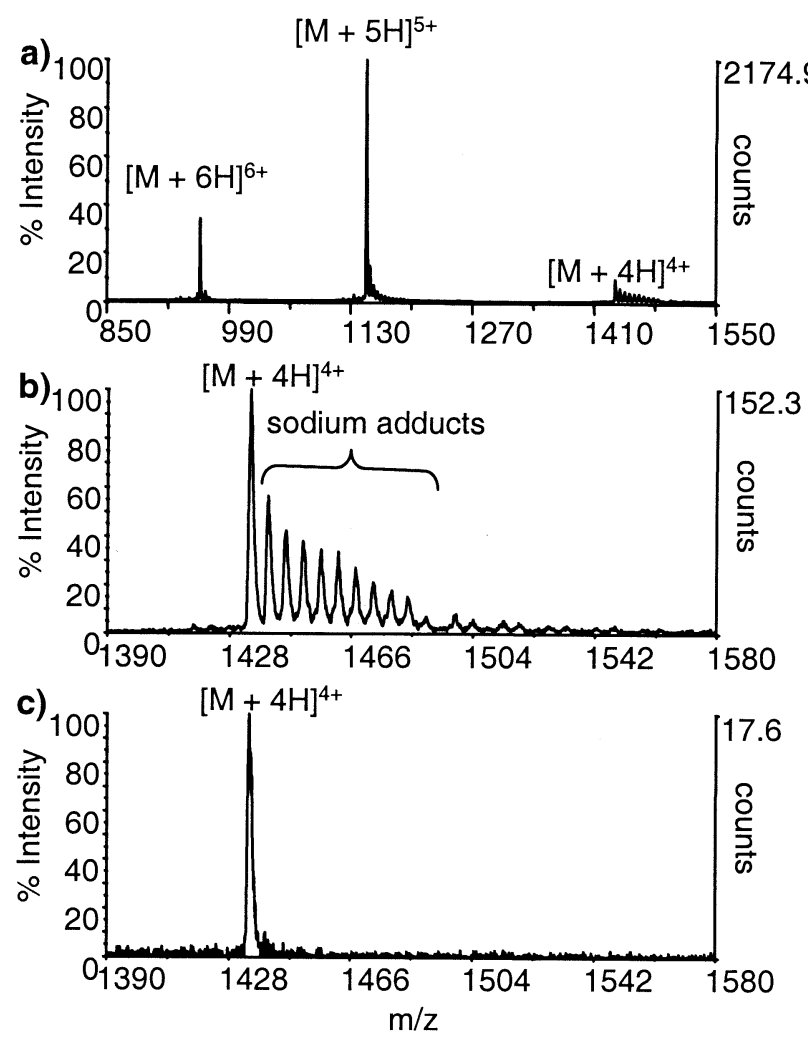

Figure 6. Mass spectra of insulin $\left(\mathrm{c}=10^{-5} \mathrm{~mol} / \mathrm{L}\right)$ in methanol/ water $/ 4 \%$ acetic acid, $10 \mathrm{mM}$ sodium chloride. (a) Tip opening o.d. $\approx 10 \mu \mathrm{m}, \mathrm{I}_{\text {Spray }}=60 \mathrm{nA} ;(\mathbf{b})$ charge state +4 enlarged from Figure $6 \mathrm{a}$; (c) charge state +4 , tip opening o.d. $1 \mu \mathrm{m}, \mathrm{I}_{\text {Spray }} \approx 1 \mathrm{nA}$.

flow rate the intensity is lower by a factor of $\sim 9$. However, the spray current is also lower by a factor of $\sim 60$ and therefore the quotient [signal intensity]/[spray current], i.e., the relative signal intensity, is clearly higher for the low flow rate case.

\section{Barium Bromide as a Probe for Initial Droplet Size?}

Since there are only theoretical predictions of the initial droplet size for different flow rates, a simple model system was investigated in order to qualitatively connect the ion signal observed in nano-ESI mass spectra to the initial droplet size. Electrolytes such as salts are suitable compounds for this purpose and the main characteristics of the mass spectra produced from these species have been studied extensively by ESI MS [29]. In positive-ion mode ESI MS, barium bromide is typically detected as $\mathrm{Ba}^{2+}, \mathrm{Ba}^{+}, \mathrm{BaBr}^{+}$, and several cluster ions resulting from gas-phase reactions with the solvent which have been described previously for other divalent cations [30-33]. For a given solvent, the signal intensity of the ion pair $\mathrm{BaBr}^{+}$in comparison to $\mathrm{Ba}^{2+}$ and $\mathrm{Ba}^{+}$increases with increasing concentration of $\mathrm{BaBr}_{2}$ in solution, because the increased ion-pair formation in solution is reflected in the mass spectra [34, 35]. For a given analyte concentration, the relative intensity of $\mathrm{BaBr}^{+}$in comparison to $\mathrm{Ba}^{2+}$ and $\mathrm{Ba}^{+}$reflects 


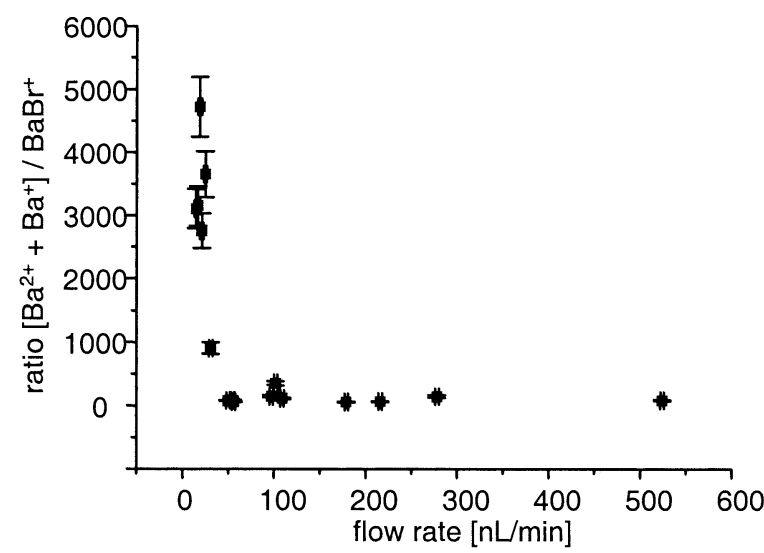

Figure 7. Signal intensity ratio $\left[\mathrm{Ba}^{2+}+\mathrm{Ba}^{+}\right] /\left[\mathrm{BaBr}^{+}\right]$observed for $\mathrm{BaBr}_{2}$ solutions, $\mathrm{c}=10^{-4} \mathrm{~mol} / \mathrm{L}$ in ethanol at different liquid flow rates.

changes in the initial droplet size because larger initial droplets require more solvent evaporation throughout the droplet fission cascade and prior to ion release, and yield a higher ratio of ion-pair formation. Figure 7 shows the intensity ratio $\left[\mathrm{Ba}^{2+}+\mathrm{Ba}^{+}\right] /\left[\mathrm{BaBr}^{+}\right]$obtained for different liquid flow rates. Only at low flow rates is a high intensity ratio (i.e., low intensity of the $\mathrm{BaBr}^{+}$ion pair) observed.

However, when interpreting the data from Figure 7, one should be aware of the fact that the above considerations only apply as long as the number of anions that is removed from the solution by the electrochemical charging process is negligible compared to the number of anions available in the solution. This condition is usually met at conventional ESI conditions, but caution is needed either for spray of low salt concentrations or at low liquid flow rates, as outlined above. At the extreme, the spray current could exclusively be attributed to the discharge of the anions present in the solution; all of the anions would be removed electrochemically from a $10^{-4} \mathrm{~mol} / \mathrm{L} \mathrm{BaBr}_{2}$ solution when taking into account the data shown in Table 2. However, it is typically assumed that also solvent oxidation and emitter corrosion contribute to the spray current. Thus, with the above considerations it is questionable whether the $\mathrm{Ba}^{2+} / \mathrm{BaBr}^{+}$intensity ratio can be regarded as a direct probe for the initial droplet size. Nevertheless, the ratio is characteristic for the spray process at different flow rates.

\section{Conclusion}

The flow rates in nano-ESI exhibit a strong influence on ion signals. Analyte suppression can be completely absent even for analytes of strongly different physicochemical properties and occurs quite abruptly once the region of very small flow rates is left. The suppression effects are already pronounced at flow rates in the range of $50 \mathrm{~nL} / \mathrm{min}$. Consequently, even though these "lowflow" spray conditions still exhibit the advantage of low sample consumption, other features of "true" nano-ESI are no longer present under these conditions. This issue should also be kept in mind for nano-LC MS applications which usually operate in this regime of liquid flow rates. The proposed and used analyte test mixtures provide a simple means to specify the actual ESI conditions used in any experiment.

The practical consequences depend on the intention of an analysis: If the absence of any suppression effects is required (i.e., for mixture analysis or in the presence of compounds with higher surface activity than the analyte), the flow rate should be as low as possible, and the use of nano-ESI needles with small tip diameters is recommended. However, the adverse effect, i.e., the preference for charged compounds exhibiting some surface activity - a typical peptide with basic functional groups at low $\mathrm{pH}-$ can also be understood in the context of this discussion and rationalizes the success of ESI, even at $\mu \mathrm{L}$ flow rates.

\section{References}

1. Aleksandrov, M. L.; Gall, L. N.; Krasnov, N. V.; Nikolaev, V. I.; Pavlenko, V. A.; Shkurov, V. A. Extraction of Ions from Solutions at Atmospheric Pressure-Method of Mass Spectrometric Analysis of Bioorganic Compounds. Dok. Akad. Nauk SSSR 1984, 277, 379-383.

2. Yamashita, M.; Fenn, J. B. Electrospray Ion Source: Another Variation on the Free-Jet Theme. J. Phys. Chem. 1984, 88, 4451-4459.

3. Dole, M.; Mack, L. L.; Hines, R. L.; Mobley, R. C.; Ferguson, L. D.; Alice, M. B. Molecular Beams of Macroions. J. Chem. Phys. 1968, 49, 2240-2249.

4. McLafferty, F. W.; Fridriksson, E. K.; Horn, D. M.; Lewis, M. A.; Zubarev, R. A. Biomolecule Mass Spectrometry. Science 1999, 284, 1289-1290.

5. Bakhtiar, R.; Nelson, R. W. Electrospray Ionization and Matrix-Assisted Laser Desorption Ionization Mass Spectrometry. Biochem. Pharmacol. 2000, 59, 891-905.

6. Hofstadler, S. A.; Griffey, R. H. Analysis of Noncovalent Complexes of DNA and RNA by Mass Spectrometry. Chem. Rev. 2001, 101, 377-390.

7. Aebersold, R.; Goodlett, D. R. Mass Spectrometry in Proteomics. Chem. Rev. 2001, 101, 269-295.

8. Tang, L.; Kebarle, P. Dependence of Ion Intensity in Electrospray Mass Spectrometry on the Concentration of the Analytes in the Electrosprayed Solution. Anal. Chem. 1993, 65, 36543668.

9. Wilm, M. S.; Mann, M. Electrospray and Taylor-Cone Theory, Dole's Beam of Macromolecules at Last? Int. J. Mass Spectrom. Ion Processes 1994, 136, 167-180.

10. Wilm, M. S.; Mann, M. Analytical Properties of the Nanoelectrospray Ion Source. Anal. Chem. 1996, 68, 1-8.

11. Bahr, U.; Pfenninger, A.; Karas, M.; Stahl, B. High Sensitivity Analysis of Neutral Underivatized Oligosaccarides by NanoElectrospray Mass Spectrometry. Anal. Chem. 1997, 69, 45304535.

12. Juraschek, R.; Dülcks, T.; Karas, M. Nanoelectrospray-More Than Just a Minimized-Flow Electrospray Ionization Source. J. Am. Soc. Mass Spectrom. 1999, 10, 300-308.

13. Fernandez de la Mora, J.; Loscertales, I. G. The Current Emitted by Highly Conducting Taylor Cones. J. Fluid Mech. 1994, 260, 155-184.

14. Pfeifer, R. J.; Hendricks, C. D., Jr. Parametric Studies of Electrohydrodynamic Spraying. AIAA J. 1968, 6, 496-502. 
15. Enke, C. G. A Predictive Model for Matrix and Analyte Effects in Electrospray Ionization of Singly-Charged Ionic Analytes. Anal. Chem. 1997, 69, 4885-4893.

16. Cech, N. B.; Enke, C. G. Relating Electrospray Ionization Response to Nonpolar Character of Small Peptides. Anal. Chem. 2000, 72, 2717-2723.

17. Constantopoulos, T. L.; Jackson, G. S.; Enke, C. G. Challenges in Achieving a Fundamental Model for ESI. Anal. Chim. Acta 2000, 406, 37-52.

18. Tang, K.; Smith, R. D. Physical/Chemical Separations in the Breakup of Highly Charged Droplets from Electrosprays. J. Am. Soc. Mass Spectrom. 2001, 12, 343-347.

19. Schmidt, A.; Bahr, U.; Karas, M. The Influence of Pressure in the First Pumping Stage on Analyte Desolvation and Fragmentation in Nano-ESI MS. Anal. Chem. 2001, 73, 6040-6046.

20. Raffaelli, A.; Bruins, A. P. Factors Affecting the Ionization Efficiency of Quarternary Ammonium Compounds in Electrospray/Ionspray Mass Spectrometry. Rapid Commun. Mass Spectrom. 1991, 5, 269-275.

21. Lax, E., Ed.; D'Ans Lax Taschenbuch für Chemiker und Physiker. Springer Verlag: Berlin, 1967; p 818

22. Conway, B. E., Ed.; Elektrochemische Tabellen. Govi Verlag GmbH: Frankfurt, 1957; p 45.

23. Weast, R. C., Ed.; Handbook of Chemistry and Physics. CRC Press: Cleveland, 1974; p F-42.

24. Kebarle, P.; Ho, Y. On the Mechanism of Electrospray Mass Spectrometry. Electrospray Ionization Mass Spectrometry: Fundamentals, Instrumentation, and Applications; In: Cole, R. B., Ed.; John Wiley and Sons, Inc: New York, 1997; p 3.

25. Van Berkel, G. J. The Electrolytic Nature of Electrospray. Electrospray Ionization Mass Spectrometry: Fundamentals, Instrumentation, and Applications; In: Cole, R. B., Ed.; John Wiley and Sons, Inc: New York, 1997; p 65.

26. Van Berkel, G. J. Insight into Analyte Electrolysis in an Electrospray Emitter from Chronopotentiometry Experiments and Mass Transport Calculations. J. Am. Soc. Mass Spectrom. 2000, 11, 951-960.

27. Van Berkel, G. J. Electrolytic Deposition of Metals on to the High-Voltage Contact in an Electrospray Emitter: Implications for Gas-Phase Ion Formation. J. Mass Spectrom. 2000, 35, 773-783.

28. Zhou, S.; Cook, K. D. A Mechanistic Study of Electrospray Mass Spectrometry: Charge Gradients within Electrospray Droplets and Their Influence on Ion Response. J. Am. Soc. Mass Spectrom. 2001, 12, 206-214.

29. Colton, R. D'; Agostino, A.; Traeger, J. C. Electrospray Mass Spectrometry Applied to Inorganic and Organometallic Chemistry. Mass Spectrom. Rev. 1995, 14, 79-106.

30. Blades, A. T.; Jayaweera, P.; Ikonomou, M. G.; Kebarle, P. Studies of Alkaline Earth and Transition Metal++ Gas Phase Ion Chemistry. J. Chem. Phys. 1990, 92, 5900-5906.

31. Kohler, M.; Leary, J. A. Gas-Phase Reactions of Doubly Charged Alkaline Earth and Transition Metal Complexes of Acetonitrile, Pyridine, and Methanol Generated by Electrospray Ionization. J. Am. Soc. Mass Spectrom. 1997, 8, 1124-1133.

32. Kohler, M.; Leary, J. A. Gas Phase Reactions of Doubly Charged Alkaline Earth and Transition Metal(II)-Ligand Complexes Generated by Electrospray Ionization. Int. J. Mass Spectrom. Ion Processes 1997, 162, 17-34.

33. Jayaweera, P.; Blades, A. T.; Ikonomou, M. G.; Kebarle, P. Production and Study in the Gas Phase of Multiply Charged Solvated or Coordinated Metal Ions. J. Am. Chem. Soc. 1990, $112,2452-2454$.

34. Wang, G.; Cole, R. B. Mechanistic Interpretation of the Dependence of Charge State Distribution on Analyte Concentration in Electrospray Ionization Mass Spectrometry. Anal. Chem 1995, 67, 2892-2900.

35. Wang, G.; Cole, R. B. Effects of Solvent and Counterion on Ion Pairing and Observed Charge States of Diquaternary Ammonium Salts in Electrospray Mass Spectrometry. J. Am. Soc. Mass Spectrom. 1996, 7, 1050-1058. 